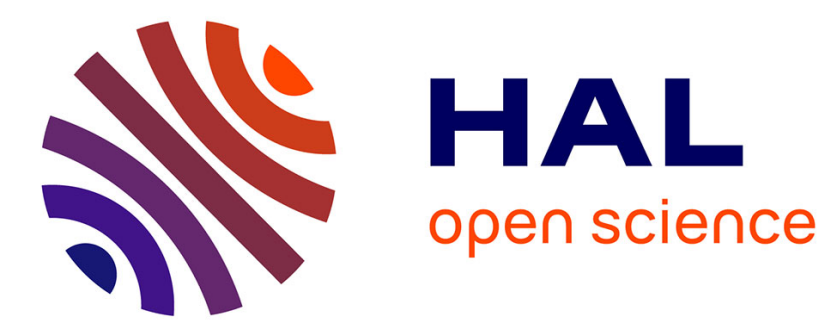

\title{
Mutations et déclin du Mouvement Pachakutik en Équateur (1996-2008)
}

\author{
Julie Massal
}

\section{To cite this version:}

Julie Massal. Mutations et déclin du Mouvement Pachakutik en Équateur (1996-2008). Problèmes d'Amérique Latine, 2009. hal-03280786

\section{HAL Id: hal-03280786 https://hal.science/hal-03280786}

Submitted on 7 Jul 2021

HAL is a multi-disciplinary open access archive for the deposit and dissemination of scientific research documents, whether they are published or not. The documents may come from teaching and research institutions in France or abroad, or from public or private research centers.
L'archive ouverte pluridisciplinaire HAL, est destinée au dépôt et à la diffusion de documents scientifiques de niveau recherche, publiés ou non, émanant des établissements d'enseignement et de recherche français ou étrangers, des laboratoires publics ou privés. 


\section{Problèmes}

D’AMÉRIQUE LATINE

\section{Printemps 2009}

\section{Ethnicité}

Beatriz Urías Horcasitas

François Bourricaud

José Luis Escalona Victoria

Julie Devineau

Julie Massal

Jean-François Véran 


\title{
SOMMAIRE
}

\author{
Dossier
}

\section{ETHNICITÉ}

Coordonné par Gilles Bataillon et Julie Devineau

Ethnicité,

Gilles BATAILLON et Julie DEVINEAU

Le pouvoir des symboles/les symboles dans le pouvoir.

Théosophie et « mayanisme » dans le Yucatán (1922-1923),

Beatriz URÍAS HORCASITAS.

Quelques caractères originaux d'une culture métisse

en Amérique latino-indienne,

François BOURRICAUD.

Invocations de l'ethnicité et imaginaire sociopolitique au Mexique, José Luis ESCALONA VICTORIA

Variations régionales: la politisation des identités ethniques

au Mexique,

Julie DEVINEAU

Mutations et déclin du Mouvement Pachakutik

en Équateur (1996-2008),

Julie MASSAL

La démocratie brésilienne à l'épreuve de la "question raciale », Jean-François VÉRAN

Résumés

Resúmenes

Abstracts. 


\title{
Mutations et déclin du Mouvement Pachakutik en ÉQuateur (1996-2008)
}

\author{
Julie MASSAL *
}

Cet article se propose de retracer l'évolution du Mouvement d'unité plurinationale nouveau-pays pachakutik (ou Mouvement Pachakutik). Ce mouvement indien est le fruit de l'alliance de la Confédération des nationalités indigènes d'Équateur (CONAIE, créée en 1986), et d'une nébuleuse d'acteurs sociaux non indiens, principalement urbains. Cette alliance ample et hétéroclite s'est tissée à la faveur de la rencontre entre la CONAIE, devenue à partir du soulèvement national indien de juin 1990 un interlocuteur permanent du pouvoir, et des acteurs sociaux qui souhaitaient réformer profondément l'État et le système politique. Le Mouvement Pachakutik propose donc, à l'origine, une substantielle réforme de la démocratie, jugée formelle et insuffisante, ainsi que la reconnaissance d'un État plurinational ${ }^{1}$.

Il émerge officiellement sur la scène politique en mai 1996, en participant aux élections présidentielle et législative, et s'affirme aussitôt comme la troisième force politique nationale. Mais dix ans plus tard, le Mouvement Pachakutik connaît un retentissant échec aux élections présidentielles d'octobre 2006. Ce parcours illustre une évolution similaire à celle d'autres mouvements de même nature ${ }^{2}$ : une rapide montée en puissance politique

\footnotetext{
* Julie Massal est professeure assistante, IEPRI, université nationale de Colombie, Bogotá.

1. Julie Massal \& Marcelo Bonilla, Los movimientos sociales en las democracias andinas, Quito, FLACSO-IFEA, 2000; Julie Massal Les mouvements indiens en Équateur. Mobilisations protestataires et démocratie, Paris \& Aix-en-Provence, Karthala-CSPC-IEP, 2005.

2. Pour un bilan de la participation politique et électorale des mouvements indiens des Andes: León T. Jorge \& al., Participación política, democracia y movimientos indigenas en los Andes, Embajada de Francia en Bolivia-IFEA-PIEB, La Paz, 2005, 181 p. Compte rendu en français par Julie Massal, dans la Revue Transcontinentales, Paris, Armand Colin, $2^{\mathrm{e}}$ semestre 2006, $\mathrm{n}^{\circ} 3$, pp. 150-151.
} 
et un gain de légitimité nationale et internationale, puis un déclin électoral irrégulier (selon qu'il s'agit de scrutins locaux ou nationaux) mais affirmé. Il s'agit donc de comprendre les mutations subies par le Mouvement Pachakutik puis son déclin électoral, en insistant tant sur les facteurs conjoncturels que structurels de son évolution. Le système politique, qui semblait avoir accepté certaines demandes indiennes en les incorporant dans la Constitution de 1998, continue pourtant de faire obstacle à une véritable remise en cause des fondements et de la répartition du pouvoir. Ceci explique en partie les difficultés rencontrées par l'un des mouvements indiens les plus crédibles d'Amérique latine parmi ceux visant à incarner une alternative politique viable à long terme ${ }^{3}$.

Soulignons néanmoins que le déclin électoral ne préjuge en rien d'un échec politique global des acteurs indiens, en Équateur ou ailleurs, car ceux-ci ont joué un rôle politique et symbolique majeur, en suscitant un débat politique à la fois sur les fondements de l'État-Nation et sur l'essence et l'exercice de la démocratie en Amérique latine. Ceci étant, leur relatif échec électoral reflète le fait que, paradoxalement, l'un des acteurs ayant participé à la redéfinition des règles du jeu en faveur d'une consolidation de la démocratie se retrouve exclu en partie ou totalement du pouvoir et des fruits du développement économique et social.

\section{LES ORIGINES D'UN MOUVEMENT NON EXCLUSIVEMENT INDIEN, FRUIT D'UNE AMPLE MOBILISATION SOCIALE}

\section{L'alliance entre la CONAIE et les acteurs urbains}

Le Mouvement Pachakutik présente l'originalité de constituer une alliance entre Indiens et Blancs-Métis (c'est ainsi que l'on nomme en Équateur les individus ne revendiquant pas d'appartenance à un groupe indien), en réunissant une nébuleuse d'acteurs sociaux: organisations écologistes; acteurs du secteur public qui souhaitent défendre leur statut et les services publics; mais aussi organisations citoyennes urbaines, acteurs universitaires, etc., tous mobilisés pour renforcer l'État et le prémunir contre un modèle de développement néolibéral jugé néfaste. Il s’agit également de réformer le système politique afin de redistribuer le pouvoir, en le décentralisant, et promouvoir, à plus long terme, une démocratie participative. Enfin, au sein du mouvement, la CONAIE prône la reconnaissance constitutionnelle d'un État plurinational et pluriculturel, c'est-à-dire qui reconnaisse officiellement la diversité culturelle et accepte l'idée de représenter politiquement les groupes indiens définis comme des "nationalités indiennes ${ }^{4}$ ».

3. Donna Lee Van Cott, «Cambio institucional y partidos étnicos en Suramérica », Análisis Político, Bogotá, IEPRI-U. Nacional, Enero-abril 2003, nº 48 pp. 26-51.

4. Au nombre d'une dizaine au moment où se produit ce débat: cf. le Projet politique de la CONAIE, Quito, 1997. Ces " nationalités » se subdivisent à leur tour en " peuples indiens », eux-mêmes organisés en communautés. 
Cette alliance entre Métis et Indiens, qui constitue non seulement une originalité mais aussi un atout, résulte de la construction progressive, au cours de la décennie 1990, de liens et d'objectifs communs entre la CONAIE et les acteurs sociaux mentionnés, qui jugent indispensable une profonde réforme politique, sociale et culturelle. Cette alliance réunit donc des acteurs indiens issus des organisations paysannes, et des mouvements sociaux principalement urbains. Les prémices d'une telle alliance se situent dans les mutations politiques de la décennie 1980, qui ont conduit au déclin des syndicats des années soixante-dix. Affaiblis par une certaine répression politique sous le gouvernement León Febrés Cordero (1984-1988), ceux-ci se sont dissous et leurs dirigeants se sont intégrés aux jeunes organisations indiennes naissantes, elles-mêmes issues des mouvements paysans. Ce transfert de dirigeants sociaux a alimenté en partie la direction des mouvements indiens et favorisé une certaine connivence (non exempte de conflits idéologiques sur la compatibilité entre lutte des classes et reconnaissance des identités indiennes), entre acteurs indiens et organisations de formation marxiste.

En outre, sur le plan économique, à partir de 1992, le modèle néolibéral, caractérisé notamment par une libéralisation du secteur bancaire, la privatisation des secteurs publics et l'affaiblissement de la sécurité sociale, suscite une résistance accrue des secteurs opposés à la privatisation des services publics et des ressources naturelles stratégiques (pétrole, eau, biodiversité). La réaction des acteurs urbains rejoint en partie la préoccupation des mouvements indiens qui, en milieu rural, sont confrontés à la privatisation des ressources naturelles et de l'accès à l'eau (1994), et à une réforme de la propriété foncière défavorable aux petits paysans ${ }^{5}$.

C'est ainsi que naît, en 1994, un front entre différentes organisations indiennes et paysannes, syndicats du secteur énergétique et divers acteurs urbains. Ce front se renforce en repoussant diverses tentatives de réforme néolibérale, notamment de la sécurité sociale, promues lors de deux référendums par le gouvernement de Sixto Durán (1992-1996). Il donne naissance, en février 1996 au Mouvement Pachakutik, initialement constitué par la CONAIE, la Coordination de mouvements sociaux (CMS) et le Mouvements des citoyens pour un nouveau pays (MCNP) issu de l'université de Cuenca et représenté par le journaliste de télévision Freddy Ehlers (mais le MCNP se détache, dès novembre 1997, du Mouvement Pachakutik).

Cette alliance évolue cependant de façon irrégulière, entre soubresauts et déclin, dans la décennie 1996-2006; et c'est lorsqu'elle faiblit que le mouvement Pachakutik décline sur le plan électoral. Divers facteurs tendent à la diviser, principalement des divergences idéologiques de fond quant à l'État plurinational, revendication qui est loin de faire l'unanimité. De fait,

5. Pour une analyse des effets du modèle néolibéral sur le mouvement indien: Deborah Yashar, "Democracy, indigenous movements and the post-liberal challenge in Latin America”, World Politics: A Quaterly Journal of International Relations, octobre 1999, n 52 , pp. 76-104. 
une profonde division perdure entre les acteurs de formation marxiste et une nouvelle génération de jeunes dirigeants indiens, davantage axés sur la nécessaire reconnaissance des identités indiennes et leur représentation politique. Ces clivages se retrouvent non seulement entre les différentes composantes de Pachakutik, mais aussi au sein des organisations locales qui composent la CONAIE.

Pour enrayer ces divisions, la direction de la CONAIE représentée par Luis Macas (1993-1997) tente d'adoucir cette requête, en revendiquant surtout un État pluriculturel, fondé sur la reconnaissance de la diversité culturelle, et en insistant moins sur les implications politiques de cette reconnaissance. La CONAIE obtient d'ailleurs gain de cause en 1998, lorsque la Constitution admet l'existence de "peuples qui s'auto-définissent comme des nationalités aux racines ancestrales » et leur octroie un certain nombre de droits collectifs (articles 83 et 84$)^{6}$. L'État plurinational n'est certes pas reconnu en tant que tel, mais la revendication majeure est cependant satisfaite, sur le papier.

\section{Le choix de la participation au sein du système politique}

La formation du mouvement Pachakutik obéit à un virage stratégique en faveur de la participation au système politique en vigueur. Ce virage est amorcé en 1993, au sein de la CONAIE, et s'il ne fait pas l'unanimité parmi les organisations indiennes locales, il est progressivement soutenu par les alliés de la CONAIE, désireux d'accéder au pouvoir et de réformer l'État. Un tel changement de stratégie est favorisé par une plus forte crédibilité de la CONAIE sur le plan politique, grâce à la capacité de mobilisation dont elle fait preuve lors des soulèvements nationaux de 1990 et 1994 et de la marche des organisations amazoniennes de 1992; trois moments de forte mobilisation à l'échelle nationale, qui octroient une réelle visibilité au mouvement social indien incarné par la CONAIE, devenue l'interlocuteur légitime du pouvoir. Cela permet à la CONAIE d'aspirer à une participation politique autonome à l'égard des partis politiques dits traditionnels, qui ont longtemps tiré profit du vote indien. Le défi est donc de créer un nouvel acteur

6. Les principaux droits collectifs (il y en quinze énumérés dans la Constitution, Titre III, chapitre V, section première, art. 84) sont: 1. Conserver la propriété imprescriptible des terres communautaires, qui seront inaliénables, incessibles et indivisibles, sauf en ce qui concerne celles pour lesquelles l'État a faculté à déclarer l'utilité publique. Ces terres seront exemptées de l'impôt foncier; 2. Participer à l'usage, l'usufruit et l'administration des ressources naturelles renouvelables qui se situent sur leurs terres; 3 . Être consultés sur les plans et programmes de prospection et d'exploitation des ressources non renouvelables qui se trouvent sur leurs terres, qui peuvent les affecter écologiquement ou culturellement; participer aux bénéfices qui seraient issus de ces programmes, dès que possible, et recevoir des indemnités pour les préjudices sociaux et écologiques qui en résulteraient; 4. Ne pas être déplacés, comme peuples, de leurs terres; 5 . Voir reconnue la propriété intellectuelle collective de leurs savoirs ancestraux; et [le droit à] la valorisation, à l'usage et au développement de ceux-ci conformément à la loi ; 6 . Accéder à une éducation de qualité. Compter avec le système d'éducation interculturelle bilingue. 
politique, susceptible de conquérir ce vote indien en le détournant des partis traditionnels, notamment de droite ou de type populiste, fort bien ancrés dans les régions où la population indienne est majoritaire (Sierra centrale et du nord). Toutes ces raisons expliquent le choix des acteurs indiens réunis au sein de la CONAIE de composer avec leurs alliés sociaux un mouvement politique nouveau pour les représenter politiquement.

\section{L'ascension irrégulière du Mouvement Pachakutik et le tournant du 21 JANVIER 2000}

\section{Une évolution électorale irrégulière}

Les élections générales de mai 1996 reflètent le succès immédiat du Mouvement Pachakutik, qui tient à deux raisons. En premier lieu, ce mouvement bénéficie de façon conjoncturelle d'un effet indéniable de nouveauté, au sein d'un système politique dominé, depuis le retour à la démocratie en 1979, par quatre partis ${ }^{7}$. Ainsi, il représente une alternative inédite, dans le contexte d'un débat sur le discrédit de la classe politique « traditionnelle ». En 1996, la campagne est dominée par deux partis, l'un de centre droit, l'autre de type populiste de droite: le Parti social chrétien (PSC) et le Parti roldosiste équatorien (PRE). Toux deux partagent une base électorale similaire (l'électorat de la Costa) et doivent conquérir, pour faire la différence, l'électorat indien de la Sierra. Face à eux, Pachakutik incarne une offre politique identifiée comme étant de gauche.

C'est la deuxième raison du succès: Pachakutik vise à mobiliser un électorat qui se sent dépourvu de représentation, et ne prétend pas s'adresser exclusivement aux populations indiennes (dont la proportion demeure difficile à évaluer, les chiffres variant de 7 à $45 \%$ selon les sources ${ }^{8}$ ), mais bien à "l'ensemble des exclus du système politique et du développement économique ». Pachakutik capte une partie importante du vote urbain blancmétis, notamment des deux villes majeures de la Sierra, Quito et Cuenca; vote qui provient d'un électorat partagé entre ceux qui émettent un vote protestataire, et les sympathisants de ce nouveau porte-parole des « sans

7. Ce sont le Parti roldosiste équatorien (PRE, 1982), le Parti social chrétien (PSC, 1951), la Gauche démocratique (ID, 1972) et la Démocratie populaire (1978).

8. Selon le recensement de 2001 , la population indienne représente $6,8 \%$ de la population totale. Les recensements se fondent sur des critères variables dans le temps; celui-ci utilise le critère de l'autodéfinition. D’autres sources (José SánchezParga, Escolarización y bilinguïsmo en la Sierra Ecuatoriana, Quito, Centro Andino de Acción Popular, 1991) évoquent le chiffre de 7,1\%, en se basant sur l'usage d'une langue indienne et l'appartenance à une communauté indienne. Une étude plus précise (León Zamosc, Estadística de las áreas de predominio étnico de la Sierra ecuatoriana: población rural, indicatores cantonales y organizaciones de base, Quito, Abya Yala, 1995) évalue la population indienne à $35 \%$ de la population totale. R. Santana (Les Indiens d'Équateur, citoyens dans l'ethnicité?, Toulouse-le Mirail, GRALCNRS, 1992) évoque le chiffre de $45 \%$. Il est donc délicat d'évaluer l'électorat indien au niveau national. 
voix ». Son candidat à l'élection présidentielle en mai 1996, Freddy Ehlers, obtient $20,6 \%$ des voix, se classant ainsi troisième, tandis que Pachakutik obtient aux élections législatives du 19 mai huit députés, devenant ainsi la quatrième force politique nationale.

Mais on constate d'ores et déjà que son assise électorale est fragile, déséquilibrée sur le plan national et même régional, centrée sur certaines villes et provinces de la Sierra. De plus, il n'a pas su capter le vote indien rural des provinces majoritairement indiennes, plutôt acquises au PRE d'Abdalá Bucaram (le vainqueur de l'élection présidentielle), deuxième force politique nationale en 1996. Ainsi, malgré le réel succès de 1996, les bases électorales de Pachakutik s'avèrent plutôt urbaines et moins indiennes qu'espéré.

L'évolution électorale s'avère par la suite irrégulière, alternant succès et échecs sur le plan local ou national. Ainsi, aux élections présidentielle et législative de 1998, le Mouvement Pachakutik décline, affaibli du fait de divisions internes et de flottements dans sa stratégie électorale ${ }^{9}$, pour devenir la cinquième force politique. Il se reconstitue toutefois lors des élections municipales et autres élections locales de 2000, puis aux élections générales de 2002. Enfin Pachakutik faiblit de nouveau lors des scrutins locaux d'octobre 2004 et aux scrutins nationaux, présidentiel et législatif, de l'automne 2006.

Cet échec est en partie lié à des erreurs sur le plan stratégique et à une modification des règles du jeu politique en faveur des partis traditionnels qui se sont renforcés. Mais la faiblesse électorale de Pachakutik est aussi due à la dispersion du vote indien. Des organisations indiennes soutiennent d'autres forces politiques, notamment d'obédience évangéliste, qui rivalisent donc avec Pachakutik dans la conquête de l'électorat indien. Ainsi, la CONAIE et Pachakutik doivent sans cesse reconquérir les populations indiennes, face à une offre politique rivale de plus en plus solide.

Cette évolution irrégulière est également liée à la capacité de pression fluctuante du Mouvement Pachakutik et de la CONAIE sur le système politique équatorien. En effet leur rôle varie, durant les principaux événements qui ont marqué la vie politique équatorienne depuis quinze ans. Ces événements sont: le tournant stratégique que constitue le coup d'État de janvier 2000, auquel participe la CONAIE; les élections de 2002 et la participation gouvernementale du Mouvement Pachakutik en 2003. Nous reviendrons sur leur impact ultérieurement. Mais indépendamment des événements, des lignes de divisions persistent entre la CONAIE (organisation sociale revendicative) et Pachakutik (mouvement politique), reflet des dissensions internes à la CONAIE. Faute d'accord sur la stratégie électorale, il en résulte

9. Pachakutik choisit sans conviction F. Ehlers comme candidat bien que ce dernier se soit distancé dès 1997 du mouvement. De plus, un ensemble de réformes électorales applicables dès 1998 favorise les partis traditionnels. Enfin, l'offre politique lors de cette élection dessine un scénario peu propice et l'effet de nouveauté s'est déjà fort atténué. 
des choix contradictoires qui finissent par ternir leur crédibilité auprès des populations indiennes.

L'évolution électorale du Mouvement Pachakutik en dents de scie ne signifie cependant pas que la CONAIE suive la même trajectoire. Celle-ci dispose d'une capacité de mobilisation sociale autonome, pas nécessairement liée à la force électorale du Mouvement Pachakutik. Raison pour laquelle il convient d'insister sur la distinction entre déclin électoral et influence politique des acteurs indiens. Le destin de la CONAIE ne se confond pas avec celui du Mouvement Pachakutik, bien qu'elle en soit l'un des principaux membres.

\section{La participation de la CONAIE au coup d'État du 21 janvier 2000}

Le coup d'État du 21 janvier 2000 constitue un tournant stratégique pour la CONAIE et affaiblit le Mouvement Pachakutik. Malgré son relatif déclin en 1998, ce dernier représente toujours un acteur de poids au sein du système politique équatorien, puisqu'il repose sur la force de mobilisation sociale de la CONAIE, particulièrement importante en 1997-2000. Le Mouvement a su conquérir une représentation législative et fait partie du système institutionnel. Il refuse donc d'appuyer la CONAIE, lors du coup d'État du 21 janvier 2000, lorsque celle-ci noue une alliance a priori insolite avec les forces armées, car cela remet en cause la stratégie de conquête du pouvoir par la voie électorale, avec pour horizon l'arrivée au pouvoir en 2006, selon l'objectif annoncé par la CONAIE. Sans analyser ici de façon détaillée les fondements d'une telle alliance et le contexte dans lequel elle a lieu ${ }^{10}$, évoquons brièvement quelques éléments qui expliquent cette alliance de la CONAIE et l'armée.

Historiquement, les forces armées, en Équateur, se veulent le représentant légitime du peuple, contre des élites conservatrices peu soucieuses de l'intérêt général, et des partis politiques jugés peu représentatifs. Néanmoins, il existe au sein de l'institution militaire deux visions distinctes du développement national, et partant, de la relation qu'il convient d'entretenir avec les acteurs indiens: répression ou intégration. Les partisans de la répression sont convaincus que l'État se confond avec la Nation; dès lors, la revendication d'État plurinational prônée par la CONAIE est assimilée à une volonté de sécession et à ce titre jugée inadmissible. Cette branche " conservatrice ", dominante, est donc plutôt hostile aux mouvements indiens qu'elle juge potentiellement subversifs. Au contraire, une branche " progressiste » de l'armée prône une option intégratrice, accepte la légitimité des revendications indiennes de l'État pluriculturel, voire de la plurinationalité, et affiche sa préoccupation pour l'ampleur de la pauvreté et la marginalisation des populations indiennes. Cette branche juge nécessaire la canalisation (et le

10. Julie Massal, Les mouvements indiens en Équateur. Mobilisations protestataires et démocratie, op.cit., chap. 8. 
contrôle) des demandes sociales indiennes, tout en développant le rôle social de l'armée auprès des populations les plus démunies.

En janvier 2000, l'armée de terre, principale protagoniste du coup d'État, obéit plutôt à la vision intégratrice. En outre, elle a de profonds motifs internes pour s'opposer au président, Jamil Mahuad (1998-2000), notamment la réduction de son budget après la signature de la paix avec le Pérou (1998), et la redéfinition de son rôle social et économique, en situation de grave crise économique (1999). Des officiers de haut rang tissent donc une alliance avec la CONAIE et les mouvements sociaux, profondément hostiles au gouvernement du fait de sa politique économique. La décision de l'armée de priver J. Mahuad de son appui intervient lorsque le président annonce la dollarisation de l'économie, le 9 janvier 2000. L'armée argue du rejet populaire massif à l'encontre de J. Mahuad ${ }^{11}$ pour lui dénier son soutien, l'obligeant à fuir. Durant quelques heures, un triumvirat incarné par Lucio Gutiérrez, colonel de l'armée de terre, Antonio Vargas, président de la CONAIE, et Carlos Solorzano, représentant des mouvements sociaux, prend le pouvoir, avant d'être à son tour contraint de le céder au vice-président Gustavo Noboa, sous la pression du Haut Commandement militaire ${ }^{12}$.

La participation de la CONAIE au coup d'État engendre un conflit avec le Mouvement Pachakutik, dont les députés, élus en 1998, refusent de démissionner comme le leur demandait la CONAIE. Les dirigeants de Pachakutik craignent un discrédit du mouvement politique indien. Toutefois Pachakutik obtient un succès électoral certain en mai 2000 aux élections municipales et provinciales, ce qui lui permet de postuler en position de force pour les élections générales en 2002.

Suite au coup d'État, des alliances entre différents officiers retirés, membres du parti créé par L. Gutiérrez (Parti société patriotique, PSP), en juillet 2000, et les acteurs indiens et sociaux, se sont tissées. Ces alliances débouchent sur un accord faisant de Lucio Gutiérrez, ex-colonel de l'armée, amnistié par le Congrès (mai 2000), le candidat du Mouvement Pachakutik aux élections générales de l'automne 2002, choix qui ne fait toutefois pas l'unanimité. Cependant, L. Gutiérrez conquiert la présidence en novembre 2002 avec $55 \%$ des voix, grâce au soutien d'une ample coalition des mouvements sociaux, de la CONAIE et Pachakutik ${ }^{13}$, et entre en fonction le 15 janvier 2003.

11. Ce rejet tient à une brutale paupérisation, fruit de la crise économique et bancaire de 1999, encore accentuée par les mesures prises pour éviter la fuite des capitaux et organiser le sauvetage des banques, telles que le gel des comptes des épargnants, pour un an d'abord, puis pour dix ans.

12. Sur les événements du 21 janvier 2000: Dossier de la Revue OSAL, CLACSO, Buenos Aires, junio de 2000, pp. 5-37.

13. Massal Julie, «Le discours de la surprise électorale en contexte de démocratisation. L'exemple des élections équatorienne et bolivienne en 2002 ", dans Olivier Dabène, Michel Hastings \& Julie Massal (dirs), La surprise électorale. Paradoxes du suffrage universel, Aix-en-Provence, Karthala-CSPC-IEP, 2007, 262 p., pp. 107-126. 


\section{La participation gouvernementale de Pachakutik en 2003 et Ses CONSÉQUENCES.}

Pachakutik est à l'origine d'une expérience inédite (antérieure à l'ascension au pouvoir d'Evo Morales en Bolivie, en décembre 2005), lorsqu'il participe au pouvoir exécutif, au sein du gouvernement de Lucio Gutiérrez, en 2003. Cette expérience qui apparaissait comme une opportunité historique pour les Indiens est pourtant un flagrant échec. Cela tient tout d'abord aux faiblesses et erreurs de Pachakutik: il perd du crédit auprès des bases en imposant des candidats peu visibles et non reconnus dans les régions indiennes, adopte des pratiques décriées par les bases dans sa façon de faire campagne, et ne dispose pas d'une base militante suffisante capable de le faire connaître en dehors des périodes de mobilisation électorale. Mais l'échec est aussi lié aux obstacles que le système politique oppose aux acteurs indiens, qui restreignent la marge de manœuvre du Mouvement Pachakutik qui, en tant que mouvement politique ${ }^{14}$, est autorisé par la loi sur les acteurs politiques indépendants (1996) à se présenter aux élections mais ne bénéficie pas des financements publics que reçoivent les partis politiques; de façon générale, les conditions d'une compétition politique égalitaire ne sont pas réunies.

\section{Les répercussions négatives de la participation au gouvernement}

Au sein du gouvernement Gutiérrez, le Mouvement Pachakutik compte six ministres ${ }^{15}$, qui n'ont pas un budget suffisant pour agir et ont peu de marge de manœuvre politique; en outre ils se trouvent bientôt en contradiction avec la politique économique suivie par L. Gutiérrez qui, renonçant à ses promesses électorales, se rapproche des États-Unis et du Fonds monétaire international, menant alors une politique économique erratique mais contradictoire avec son opposition de principe au néolibéralisme. Il en résulte de fortes divisions entre Pachakutik et L. Gutiérrez. Mais le mouvement politique tente de se maintenir au pouvoir, malgré la distance prise par la CONAIE et l'ensemble des mouvements sociaux envers L. Gutiérrez. Pourtant, le vice Premier ministre jette l'éponge en juin et deux autres ministres sont remerciés peu après. Le départ définitif de Pachakutik, en position de faiblesse et sous la contrainte, intervient le 6 août $2003^{16}$.

14. La loi en question reconnaît à la fois la possibilité pour des figures individuelles et des mouvements politiques (qui refusent de se constituer en partis) de participer aux élections en tant " qu'indépendants ». Les mouvements politiques n’obéissent pas aux règles de fonctionnement édictées par les lois sur les partis politiques depuis 1978.

15. Ce sont Virgilio Hernández, vice Premier ministre; Luis Macas, ministre de l'Agriculture, Nina Pacari, ministre des Affaires étrangères, et trois autres ministres (éducation, bien-être social, tourisme), ainsi qu'un délégué chargé de la décentralisation, Augusto Barrera.

16. Julie Massal, «La participation du Mouvement Pachakutik au pouvoir en 2003: une expérience originale de citoyenneté multiculturelle en Équateur? ", dans JeanMichel Blanquer \& al. (dir.), Voter dans les Amériques, Paris, IHEAL-La SorbonneInstitut des Amériques, 2005, pp. 263-272. 
Le mouvement indien social et politique en sort très affaibli et discrédité, faute de réaliser de véritables innovations politiques. De plus sa pratique politique et le fait qu'il soit en contradiction avec la politique économique de L. Gutiérrez le conduisent à se comporter en mouvement d'opposition. Cela ravive l'idée, chez ses adversaires, qu'il s'agit d'un acteur forgé dans la protestation de rue, mal préparé pour l'exercice du pouvoir. Enfin, Pachakutik souffre de faiblesses intrinsèques qui l'empêchent bel et bien d'exercer le pouvoir: un manque de cadres formés et aptes à prendre en charge des dossiers épineux (d'ordre économique) et un manque de rénovation de ses dirigeants.

À cela s'ajoute une coupure entre les élites dirigeantes du mouvement et les militants de base au niveau local, qui ne se sentent ni consultés ni représentés. Cette coupure, visible dès $1998^{17}$ et qui n'a fait que s'accentuer depuis, explique la faible légitimité des décisions des dirigeants indiens auprès de leurs bases. Ainsi des militants ou des membres des communautés indiennes expriment un profond scepticisme ou une indifférence face au discours de la plurinationalité, fer de lance de la CONAIE; le choix d'appuyer telle ou telle candidature électorale suscite des divisions locales. Dès lors, Pachakutik perd du crédit auprès des populations indiennes et des secteurs de population qui l'ont soutenu électoralement. Sa difficulté à incarner une alternative politique accentue le mécontentement d'une fraction de la CONAIE, opposée depuis toujours à la participation au sein du système politique ${ }^{18}$. De son côté, la CONAIE est discréditée auprès des communautés indiennes, cooptées par la politique clientéliste intensive du parti de Lucio Gutiérrez. Ce dernier ramène les populations indiennes dans le giron d'un gouvernement plus paternaliste qu'intégrateur, tout en divisant les organisations indiennes et en décrédibilisant Pachakutik.

Cette fracture du mouvement indien se reflète aux élections locales de 2004, Pachakutik réalisant un score mitigé: il obtient vingt-trois mairies, dont cinq grâce à diverses alliances locales, et quatre préfectures provinciales, dont deux en alliance ${ }^{19}$. C'est un score en régression par rapport à 2000, où il obtenait à lui seul cinq préfectures et vingt-sept mairies. Toutefois, comme le montrent les résultats électoraux depuis 1996, le problème de fond que rencontre le Mouvement Pachakutik est une représentation territoriale fort instable d'une élection à l'autre, et en l'occurrence, à la fois peu consolidée dans certaines zones de population indienne majoritaire et en voie d'effritement dans les secteurs urbains de la Sierra. Il ne dispose pas non plus d'une véritable assise électorale dans la région de la Costa. Cependant, des dynamiques locales contradictoires selon les types de scrutin

17. Entretiens à Otavalo, auprès de militants du Mouvement Pachakutik, maiaoût 2008.

18. En particulier, divers représentants de la CONAIE mais aussi de Pachakutik étaient opposés à l'alliance avec L. Gutiérrez. Entretiens conduits en août 2003 à Quito, après la rupture avec le gouvernement.

19. El Comercio, 19 octobre 2004. 
rendent complexe une évaluation nationale du mouvement social indien, qui faiblit dans certaines zones (Imbabura, Bolivar, Sucumbios) mais se fortifie ailleurs (Cotopaxi).

\section{Les obstacles opposés par le système politique}

Plus fondamentalement, les va-et-vient de la conquête du pouvoir, au niveau national mais aussi local, mettent en évidence le double défi auquel sont confrontés les acteurs indiens. Le premier est de transformer le Mouvement Pachakutik en acteur politique stable, sans toutefois se convertir en parti politique assimilable aux partis traditionnels, donc sans adopter leurs pratiques les plus décriées: l'absence de démocratie interne et l'usage du clientélisme. Or, sur le premier point, l'échec de Pachakutik est patent: les idéaux de consultation horizontale et permanente des bases se sont évanouis dès 1998; celles-ci se sentent peu prises en compte même en période de campagne électorale; enfin la faible rénovation interne des dirigeants suscite maintes critiques, parfois tacites mais non moins vives. Ainsi, Pachakutik n'est pas parvenu à incarner une alternative foncièrement distincte des partis en termes de pratiques.

Le second défi, corollaire du premier, est de se substituer durablement aux partis politiques dits traditionnels, si discrédités soient-ils, car ces derniers disposent d'une emprise sur leur électorat extrêmement forte, y compris via la coercition. Or, en tant que mouvement politique, Pachakutik dispose de moins de ressources que les partis (loi de 1996). Il n'a donc pas la possibilité de rivaliser avec la politique clientéliste des partis traditionnels. Une fois dissipée la séduction de la nouveauté, Pachakutik, discrédité par son échec gouvernemental, souffre de limites majeures qui l'empêchent de s'imposer durablement face à ses concurrents.

Bien que les mouvements indiens aient contribué par leur mobilisation, dans les décennies 1980-1990, à modifier quelque peu les règles du jeu politique et électoral, et aient pesé en faveur d'une relative démocratisation du système politique, ils n'ont pas toujours pu ou su en bénéficier. Les partis politiques traditionnels, déstabilisés en 1994, ont eux aussi mené une offensive, en 19941998, conduisant à des réformes politiques et électorales qui confirment leur prééminence. À ce contexte peu propice s'ajoutent les résultats décevants de la participation du Mouvement Pachakutik au gouvernement Gutiérrez. Cela permet de comprendre l'échec aux élections présidentielles de 2006, où le candidat du mouvement Pachakutik, Luis Macas (leader historique de la CONAIE), obtient 2,1\% des suffrages exprimés, le Mouvement Pachakutik devenant la sixième force politique nationale.

\section{DÉCLIN ÉLECTORAL, AFFAIBLISSEMENT DU MOUVEMENT SOCIAL ET MARGINALISATION POLITIQUE}

Le faible résultat électoral de Luis Macas et du Mouvement Pachakutik aux élections présidentielle et législative (il n'obtient que sept députés sur 
100) en octobre 2006 témoigne de l'effondrement de cette force politique ${ }^{20}$. Celui-ci confirme l'affaiblissement de la CONAIE en tant que mouvement social, manifeste durant la "rébellion des forajidos " qui débouche sur le renversement de Lucio Gutiérrez en avril 2005. À cette occasion, pour la première fois depuis 1990, la CONAIE joue un rôle mineur ${ }^{21}$, ne parvenant ni à se mobiliser assez tôt pour marcher vers Quito, comme elle l'avait fait lors du coup d'État de janvier 2000, ni à participer aux manifestations qui se déroulent à Quito du 13 au 20 avril ${ }^{22}$. Cette rébellion, à la différence des soulèvements indiens, qui occupent l'espace urbain mais proviennent des campagnes, est conduite par les mouvements étudiants et résulte d'une mobilisation exclusivement urbaine, principalement centrée à Quito.

Ainsi, l'affaiblissement du mouvement social indien coïncide-t-il avec son effondrement électoral, alors que ces deux dynamiques avaient évolué de façon distincte jusqu'en 2006. Le défi que rencontre Pachakutik, en tant que mouvement politique, et la CONAIE, en tant qu'organisation protestataire, est d'éviter une marginalisation définitive. Léchec de Luis Macas mériterait un examen plus détaillé. Cependant, il confirme une tendance déjà perceptible en 2002, lorsque Antonio Vargas, ex-président de la CONAIE, se présente, hors Pachakutik, et tente de capter le " vote ethnique " : il obtient tout juste 1,6\% des voix. Les tentatives d'auto-représentation des Indiens par les Indiens ont manifestement échoué. Il est cependant prématuré de conclure à l'extinction des mouvements indiens. Ceux-ci ont conquis une représentation politique qu'il est peu probable de voir aujourd'hui remise en question, mais ils doivent reconstituer leurs alliances avec d'autres acteurs sociaux.

La relation avec le nouveau gouvernement équatorien s'avère pourtant elle aussi tourmentée et témoigne du retour de la CONAIE dans l'opposition, après que celle-ci ait rompu les relations avec le président $\mathrm{R}$. Correa, en mai 2008. Si, depuis l'automne 2006, la CONAIE souhaitait une assemblée constituante dotée des pleins pouvoirs et, en ce sens, appuyait sa convocation par le président qui en avait fait son principal cheval de bataille ${ }^{23}$, elle considère néanmoins que le nouveau projet de Constitution ne présente pas de véritables innovations en faveur des peuples indiens, comparé à la Constitution de 1998. Aussi, peu avant le référendum du 28 septembre 2008 sur le nouveau texte constitutionnel, la CONAIE exprime son appui critique

20. Au sein du mouvement Pachakutik et de la CONAIE, cette analyse est partagée par certains dirigeants et militants, qui voient aussi le mouvement s'effondrer aux élections locales dans certains bastions. D'autres parlent d'un avertissement: El Comercio, 30 octobre 2006 : "La división indígena se sintió en las urnas ».

21. Comme l'admet son vice-président, Santiago de la Cruz. Quito, entretien, 28 avril 2005.

22. Sur la « Rébellion des Forajidos »: Dossier de la Revue Iconos, Quito, FlacsoEcuador, $\mathrm{n}^{\circ} 23,2005$.

23. El Comercio, 13 février 2007 (entretien avec Luis Macas sur la relation de la CONAIE avec le président Correa). Cependant, des désaccords existent entre la CONAIE et Pachakutik au sujet de l'étendue des pouvoirs de l'Assemblée: El Comercio, 21 février 2007. 
au projet officiel de Constitution élaboré par l'Assemblée, tout en soulignant que ses revendications concernant la mise en œuvre de l'État plurinational n'ont pas été prises en compte ${ }^{24}$. La Constitution est d'ailleurs approuvée par référendum et le Mouvement Pachakutik la considère comme une étape sur le chemin de l'État plurinational. Mais la CONAIE affirme ne pas soutenir Rafael Correa ${ }^{25}$, car elle est en désaccord avec sa politique minière et énergétique, du fait du refus du président de consulter les communautés indiennes quant à l'exploitation des ressources du sol et sous-sol se trouvant sur le territoire des communautés, et ce, bien que le principe de consultation ait été admis dans l'article 84 de la Constitution de 1998 au sujet des droits collectifs des peuples indiens.

Toutefois, les Indiens sont favorables à certaines des positions présidentielles sur le plan international, telles que le refus du traité de libre commerce entre l'Équateur et les États-Unis (ce traité, en négociation jusqu'aux fortes mobilisations indiennes à son encontre en juin 2006, est laissé de côté une fois R. Correa parvenu au pouvoir), et le non-renouvellement de l'accord qui permet aux États-Unis de disposer de la base militaire de Manta pour lutter contre le narcotrafic en Colombie (l'accord expire en 2009).

En dépit de ces convergences, et bien qu'il soit encore un peu tôt pour en juger, la présence au pouvoir d'un président pourtant considéré, au niveau international, comme proche des projets de gauche les plus radicaux (ceux d'Hugo Chávez au Venezuela et d'Evo Morales en Bolivie) et partisan du socialisme du XXI ${ }^{\mathrm{e}}$ siècle, ne semble guère favorable à la reconstitution du projet politique indien du Mouvement Pachakutik, du fait du démantèlement de la CONAIE depuis 2003. Cependant, d'autres organisations indiennes (notamment la Fédération nationale des organisations paysannes, indigènes et noires d'Équateur, FENOCIN, et la Fédération évangélique indienne et noire d'Équateur, la FEINE), rivales de la CONAIE, s'affirment au plan national et leur rôle politique devra faire l'objet d'études attentives.

\section{CONCLUSION}

La conquête par les acteurs indiens du pouvoir politique et des espaces institutionnels de représentation illustre une ascension rapide puis un net affaiblissement des mouvements indiens représentés par la CONAIE, le point de rupture se situant en 2003, avec la décevante expérience de participation au pouvoir du Mouvement Pachakutik, durant le gouvernement Gutiérrez.

24. Les propositions du Mouvement Pachakutik sont consultables en ligne, en particulier " Pachakutik: base idéologique et Assemblée Nationale Constituante (document interne) »http://www.pachakutik.org.ec/home/contenidos.php?id=118 \&identificaArticulo=193

25. "Indígenas de Ecuador apoyan proyecto de Constitución pero no a Correa », Univision, 5 septembre 2008; "La CONAIE frente al referendum y la nueva Constitución », CONAIE, Boletín de prensa, Quito, 4-09-08. Articles disponibles sur: http://argentina.indymedia.org/news/2008/09/624077.php 
Cette évolution montre qu'en dépit des avancées notables obtenues sur le plan politique et symbolique, les peuples indiens d'Équateur, dotés depuis 1998 d'une reconnaissance constitutionnelle, demeurent encore largement exclus du pouvoir.

Le succès indéniable des acteurs sociaux et politiques indiens d'Équateur est d'avoir su en finir avec l'image bien ancrée de peuples indiens venus d'un autre âge et voués à l'extinction, ou celle tout aussi répandue de groupes " primitifs » luttant vainement pour la survie d'une culture menacée. Les mouvements indiens sont des acteurs sociaux mobilisés, en tant que " peuples et nationalités ", d'une part pour la défense et la réinvention politique d'une identité culturelle différenciée, d'autre part pour acquérir une reconnaissance sociale et politique, dans une société en mutation où les Indiens ne sont plus exclusivement paysans et ruraux. Cependant, force est de constater que leur capacité de représentation politique n'est pas suffisante pour mener à bien les réformes indispensables à un changement en profondeur du rôle social et politique accordé aux Indiens.

Dans le même temps, la force des acteurs indiens est d'avoir rallié à leur cause des acteurs non indiens, autour d'un projet politique plus ample, visant à modifier les fondements de la citoyenneté et de l'exercice de la démocratie. Ce projet n'a sans doute pas été mis en œuvre de façon intégrale, mais il a suscité un débat politique de fond dont l'impact sur la société laisse des traces indélébiles.

Toutefois les deux difficultés majeures que rencontrent les acteurs indiens sont d'une part, une divergence permanente sur la stratégie de conquête du pouvoir, et d'autre part, le fait que progresse un certain dépit face à l'échec des différentes tentatives menées pour réformer le système politique ${ }^{26}$. Le mouvement Pachakutik se heurte à la forte résistance au changement des partis traditionnels et du parti créé par L. Gutiérrez, qui maintiennent leur emprise sur l'électorat indien via une politique clientéliste. Ainsi, les mouvements indiens ont perdu l'espace institutionnel autonome conquis durant la décennie 1990, face à un système de partis peu disposés à accepter une refonte des règles du jeu. Mais ce n'est pas là l'unique raison de leur échec.

Si les mouvements sociaux et indiens (échaudés par l'alliance fatale avec L. Gutiérrez et désormais peu portés à nouer des alliances avec d'autres figures « indépendantes » non indiennes), n'ont pas souhaité se positionner comme de véritables soutiens d'un gouvernement dit de la "nouvelle gauche ", ou s'avèrent peu capables d'exercer une pression politique réelle sur celui-ci, c'est aussi en grande partie du fait de leurs difficultés internes. La CONAIE est de plus en plus coupée de ses bases, discréditée et toujours en proie à des dissensions internes. Outre cette coupure avec leurs bases, la

26. Julie Massal, « Ecuador: la reforma política en un callejón sin salida », Análisis Político, Bogotá, IEPRI-U. Nacional, janvier-avril 2006, n 56, pp. 132-150. 
CONAIE et Pachakutik ont aussi perdu leur capacité à souder des alliances autour d'eux, refusant de partager le pouvoir et de constituer des alliances avec d'autres organisations indiennes, et tendent ainsi à s'auto-marginaliser politiquement. Le discrédit et la faiblesse actuels de l'organisation sociale (CONAIE) qui a perdu sa capacité de mobilisation contestataire, et de son représentant politique (Pachakutik) qui n'a pas su capter durablement le vote indien, sont le fruit de tactiques contradictoires, et d'une stratégie qui privilégie la conquête du pouvoir au lieu de la consolidation des bases sociales. En outre, ladite consolidation a été minée par la cooptation de dirigeants sociaux de la CONAIE et Pachakutik, aussi bien par l'État que par des ONG de développement qui ont accaparé les cadres des organisations et détourné ceux-ci vers la gestion de projets de développement locaux au détriment de projets nationaux ${ }^{27}$.

Mais le point de rupture est aussi atteint parce que les demandes des acteurs indiens de participation à la définition des politiques de gestion de l'énergie et des ressources suscitent de profonds conflits y compris avec leurs alliés supposés, peu disposés à mettre en œuvre concrètement les droits collectifs des peuples indiens et à les considérer comme de véritables interlocuteurs politiques.

27. Julie Massal, « La participation du Mouvement Pachakutik au pouvoir en 2003: une expérience originale de citoyenneté multiculturelle en Équateur? », op. cit. 


\section{CHOISEUL ÉDITIONS \\ Problèmes d'AmérIQUe latine}

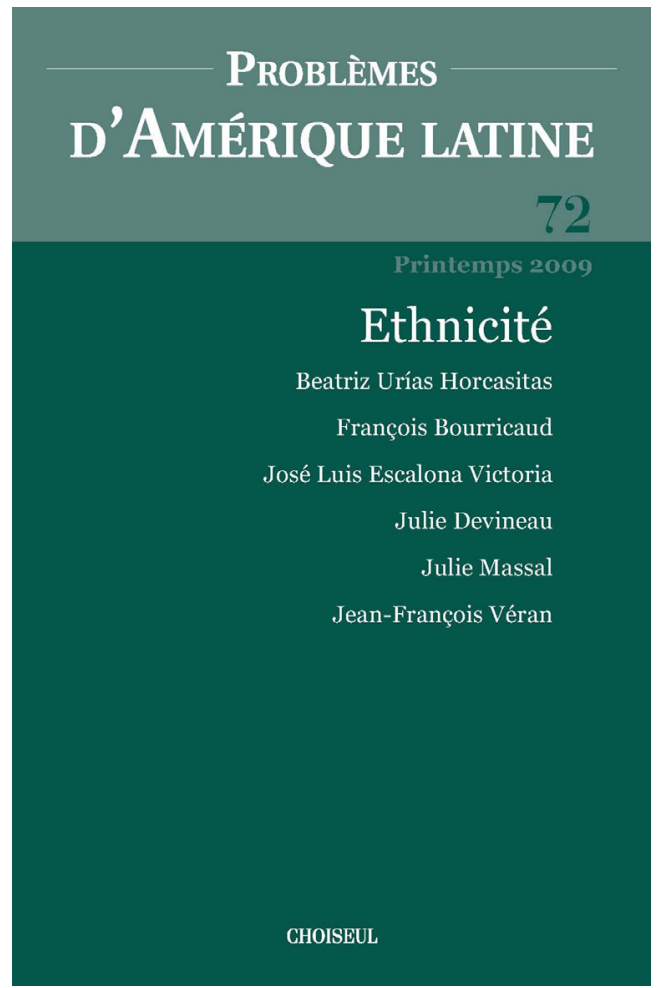

Revue trimestrielle

128 pages | $155 \times 240$

20 euros $T T C$

Achat en ligne sur www.choiseul-editions.com (paiement sécurisé)

\section{DOSSIER | \\ ETHNICITÉ}

Les revendications identitaires et indigènes ne sont pas nouvelles dans les pays d'Amérique latine. Les populations marginalisées, exclues et victimes de discriminations sociales et politiques sont essentiellement celles qui, historiquement, ont été dominées - indiens et noirs du continent.

Ce qui frappe aujourd'hui en revanche, c'est la structuration des communautés, leur capacité de mobilisation, leur visibilité médiatique et leur accession récente aux postes de pouvoir. Les politiques publiques sont appliquées, avec plus ou moins de succès, à leur avantage. Depuis une vingtaine d'années, se mettent en place de nouveaux cadres législatifs qui reconnaissent la composition multiethnique des nations. Ce qui est en jeu, c'est la structuration de la scène publique et l'apparition d'une conception ethnique de l'Etat.

Ce numéro de Problèmes d'Amérique latine s'attache à restituer et analyser en profondeur l'application de politiques publiques en faveur de l'ethnicité dans les pays latino-américains.

\section{>> DERNIERS DOSSIERS}

\section{Problėmes d'AmÉRIQue LATINE $n^{\circ} 71$ | Mutations des gauches latino-américaines}

Si l'apparition de régimes démocratiques dans les années 1980 a pour une part battu en brèche ces schémas anti-démocratiques, ceux-ci n'ont pas pour autant disparus. Nul doute que des gauches démocratiques soient apparues au Brésil ou au Chili. Au Mexique et en Argentine, tout en acceptant le verdict des élections, les gauches n'en revendiquent pas moins les héritages des populismes " historiques " ou prétendent réinventer le socialisme du XXI ${ }^{\circ}$ siècle comme au Venézuela et au Nicaragua. Cette livraison de Problèmes d'Amérique latine examine en profondeur l'évolution politique et sociale des mouvements de gauche en Amérique latine

\section{Problèmes d'Amérioue latine $n^{\circ} 70$ | Conflits et environnement}

Ce dossier met en évidence la complexité des conflits qui ont surgi sur le continent, dans lesquels l'argument environnemental occupe une place centrale - lutte pour la terre et contre la contamination, défense de la biodiversité et du cadre de vie -, mais où se joue "quelque chose de plus " : les controverses et luttes regroupées sous l'étiquette " conflits environnementaux " recouvrent en réalité d'autres demandes.

\section{Problèmes d'AmérIQue Latine $n^{\circ} 69$ | Bolivie}

La Bolivie symbolisa des années 1960 au début des années 1980 une forme d'instabilité politique propre aux régimes politiques latino-américains. Le jeu des coups de force et arrangements au sommet entre militaires et politiques prit fin en 1982 avec la mise en place d'un régime démocratico-libéral. Ce numéro d'été revient sur la remise en question du rôle central du suffrage universel, sur la situation du gouvernement d'Evo Morales, ainsi que sur les mutations qui affectent la Bolivie ces dernières années. 


\section{PROBLÈMES D'AMÉRIQUE LATINE}

BULLETIN D'ACHAT OU D'ABONNEMENT

M, Mme, Mlle Prénom

Société/Institution

$\mathrm{N}^{\circ}$ Rue

Code postal

Ville Pays

Adresse électronique

\begin{tabular}{|c|c|c|}
\hline & France & Autres pays \\
\hline 1 an (4 numéros) & $75 €$ & $85 €$ \\
\hline 2 ans (8 numéros) & $140 €$ & $160 €$ \\
\hline
\end{tabular}

Je souscris un abonnement pour

$\square 1$ an

$\square 2$ ans

À partir du numéro

Je souhaite acquérir un numéro de

Problèmes d'Amérique latine :

Date

Signature/Cachet

Paiement par virement bancaire vers

Crédit du Nord

59, boulevard Haussmann

75361 Paris, cedex 08

Banque : 30076 - Agence : 02019

Compte : 57336700200 - clé RIB : 09

IBAN : FR76 30076020195733670020009

swift (BIC) : NORDFRPP

précisez

"frais bancaires à la charge du donneur d'ordre "

ou

Paiement par chèque à l'ordre de

PROBLĖMES D'AMÉRIQUE LATINE.

Choiseul Éditions, 28 rue Étienne Marcel,

75002 Paris, France

Tel +33153340993

Attention,

les chèques étrangers doivent être en euros,

compensables en France.

$\begin{array}{ll}\square n^{\circ} 69 & \square n^{\circ} 70 \\ \square n^{\circ} 71 & \square n^{\circ} 72\end{array}$

$n^{\circ} 72$ x $20 €$ le numéro

et frais de port :

France : $2,10 €$

Reste du monde : 4, $50 €$

\section{Problèmes}

\section{D'AMÉRIQUE LATINE}

Printemps 2009

\section{Ethnicité}

Beatriz Urías Horcasitas

François Bourricaud

José Luis Escalona Victoria

Julie Devineau

Julie Massal

Jean-François Véran 